\title{
Proton transport model in the ionosphere. 2. Influence of magnetic mirroring and collisions on the angular redistribution in a proton beam
}

\author{
M. Galand ${ }^{1}$, J. Lilensten ${ }^{2}$, W. Kofman ${ }^{2}$, D. Lummerzheim ${ }^{3}$ \\ ${ }^{1}$ High Altitude Observatory, National Center for Atmospheric Research, Boulder, Colorado, USA \\ Fax: + 1303497 1589; e-mail: galand@hao.ucar.edu \\ ${ }^{2}$ Centre d'Etudes des Phénomènes Aléatoires et Géophysiques, St Martin d'Hères, France \\ ${ }^{3}$ Geophysical Institute, University of Alaska, Fairbanks, Alaska, USA
}

Received: 17 November 1997 / Revised: 6 February 1998 / Accepted: 10 February 1998

\begin{abstract}
We investigate the influence of magnetic mirroring and elastic and inelastic scattering on the angular redistribution in a proton/hydrogen beam by using a transport code in comparison with observations. $\mathrm{H}$-emission Doppler profiles viewed in the magnetic zenith exhibit a red-shifted component which is indicative of upward fluxes. In order to determine the origin of this red shift, we evaluate the influence of two angular redistribution sources which are included in our proton/ hydrogen transport model. Even though it generates an upward flux, the redistribution due to magnetic mirroring effect is not sufficient to explain the red shift. On the other hand, the collisional angular scattering induces a much more significant red shift in the lower atmosphere. The red shift due to collisions is produced by $<1-\mathrm{keV}$ protons and is so small as to require an instrumental bandwidth $<0.2 \mathrm{~nm}$. This explains the absence of measured upward proton/hydrogen fluxes in the Proton I rocket data because no useable data concerning protons $<1 \mathrm{keV}$ are available. At the same time, our model agrees with measured ground-based $\mathrm{H}$-emission Doppler profiles and suggests that previously reported red shift observations were due mostly to instrumental bandwidth broadening of the profile. Our results suggest that Doppler profile measurements with higher spectral resolution may enable us to quantify better the angular scattering in proton aurora.
\end{abstract}

Key words. Auroral ionosphere $\cdot$ Particle precipitation

\section{Introduction}

Historically, the existence of proton precipitation in the auroral regions was first inferred from ground-based

Correspondence to: M. Galand observations of Doppler-shifted hydrogen Balmer series emissions (Vegard, 1948). These $\mathrm{H}$ emissions are produced by energetic $\mathrm{H}$ atoms excited through collisions with ambient neutrals and come from the neutralization of energetic protons precipitating from the magnetosphere into the ionosphere at high latitudes. Balmer emissions, mainly $H_{\alpha}\left[\lambda_{0}=656.3 \mathrm{~nm}\right]$ but also $H_{\beta}\left[\lambda_{0}=486.1 \mathrm{~nm}\right]$, have enough intensity to be detected from the ground (Eather, 1967). H atoms emit other lines such as those of the Lyman series, but those are absorbed in the atmosphere: they are only detectable from space.

Since its discovery, the proton precipitation has been confirmed by rocket or satellite observations (e.g., Sharp et al., 1969; McNeal and Birely, 1973) and has been extensively studied (e.g., Eather, 1967; Rees, 1982; Basu et al., 1993; Kozelov and Ivanov, 1994; Decker et al., 1996). Today, energy degradation of an incident proton beam, due to collisions with atmospheric neutrals, is relatively well understood, as attested by comparisons between observations and models (e.g., Basu et al., 1987; Galand et al., 1997). A next step is now to focus on the possible angular redistribution the proton beam could undergo. For such a study, rocket measurements of particle flux would be valuable. Unfortunately, there is only one experiment which answers our needs: during the Proton I flight, particle fluxes were acquired at different altitudes, energies, and pitch angles (Söraas et al., 1974, 1994), but the data displayed no significant upward flux.

In face of the small number of particle flux observations we must look to other typical signatures of proton precipitation, such as the optical emissions in the Balmer series, $H_{\alpha}$ and $H_{\beta}$, which provide information about proton precipitation, even during the presence of electron precipitation, which is rather common. Observations of quantities relevant to $H_{\alpha}$ and $H_{\beta}$ emissions, such as their intensities measured from ground or on board rockets, have motivated many studies (Eather, 1967; Miller and Shepherd, 1969; Rees, 1982; Söraas et al., 1974; Sivjee and Hultqvist, 1975; Sigernes et al., 
1994a, b). All these studies deal with absolute intensities or altitude profiles of the hydrogen emissions. Because they combine the contributions of the large downward flux and the possible small upward flux, the angular redistribution influence on these quantities is likely to be unmeasurable. This conclusion has been confirmed by a theoretical study using a Monte Carlo method. The energy deposition function, another integrated quantity, is not significantly affected by angular redistribution of collisional (Kozelov and Ivanov, 1992) as well as of magnetic origin (Kozelov, 1993).

Other studies have focused on other characteristic quantities of the $H_{\alpha}$ and $H_{\beta}$ emissions, namely the Doppler profiles. The line profiles recorded from the ground looking along the magnetic field lines - called magnetic-zenith spectral profiles - are of particular interest. The contributions of the downward flux and the upward flux can be separated: the part of the profile related to wavelengths shorter than the characteristic wavelength $\lambda_{0}$ of the line originates from downwardmoving particles, whereas the other part comes from upward-moving particles. As most of the $\mathrm{H}$ atoms are going downward, such a Doppler profile is blue shifted (Eather, 1967). The greater part of the theoretical studies dealing with Doppler profiles of $\mathrm{H}$ emissions focus on this blue-shifted peak and take into account only the energy degradation (Eather, 1967, and references therein; Lorentzen et al., 1993; Söraas et al., 1994; Sigernes, 1996). Nevertheless, measured profiles in the magnetic zenith extend to longer wavelengths than can be explained by instrumental bandwidth (Eather, 1966). This spreading of the profiles at wavelengths higher than the characteristic wavelength $\lambda_{0}$ is called "red shift". Such a red shift, if it is not of instrumental origin, indicates that an upward flux is generated inside the proton beam and that angular redistribution sources act upon the proton beam.

Bagariatskii $(1958 \mathrm{a}, \mathrm{b})$ suggested that the red shift may be due to radiation from protons that have been magnetically reflected and are returning back up the field lines. Through capture reactions with ambient neutrals, these upward protons can then be neutralized and upward $\mathrm{H}$ atoms are generated, inducing the red shift. However, the profile calculated by Bagariatskii (1958a) for monoenergetic protons and a pitch-angle distribution proportional to $|\cos \theta|$, with $\theta$ the pitch angle, greater than $90^{\circ}$ for downward particles, showed that this magnetic effect can give only an insignificant contribution to the red shift. Later, Eather (1966) showed that the red shift might still be due to protons reflected upward by magnetic mirroring effect. Nevertheless, it was necessary to assume an initial pitch-angle distribution proportional to $|1 / \cos \theta|$ to explain the observed red shift. But such a distribution results in a Doppler shift of the zenith-profile peak that is too small. Eather (1966) concluded that, at least in the main height interval of hydrogen emission, the pitch-angle distribution must be peaked around $90-110^{\circ}$, resulting in the observed red shift; for lower pitch angles, the distributions must be, on the average, isotropic, in order to match the observed Doppler blue shift. However, satellite and rocket measurements have shown that generally the pitch angle distributions of incident downward flux are roughly isotropic from 1 to several hundreds of $\mathrm{keV}$ in diffuse aurora (Hultqvist, 1979; Basu et al., 1987) as well as in auroral arcs (Urban, 1981). Therefore, even though its action seems negligible, no definite conclusion can be drawn to date about the influence of the magnetic mirroring effect on the red shift.

Another angular redistribution source could be at the origin of the Doppler red shift observed on magneticzenith profiles of $\mathrm{H}$ emissions. In the region located between 80 and $200 \mathrm{~km}$ where most of the $\mathrm{H}$ emissions originate, the mean free path of protons and $\mathrm{H}$ atoms varies from several tens of centimeters to several kilometers. Therefore, though the angular redistribution undergone by an energetic particle through a collision with a neutral species is rather small, often lower than $1^{\circ}$ (Fleischmann et al., 1967, 1974; Cisneros et al., 1976; Van Zyl et al., 1978; Newman et al., 1986; Johnson et al., 1988; Gao et al., 1990), the variation of the pitch angle of this particle through multiple collisions at low altitude could be significant. The collisional angular redistribution could contribute greatly to the red shift. However, only Kozelov and Ivanov (1992) have theoretically studied the influence of this angular redistribution source on a proton beam, but they have not estimated its contribution to the red shift.

Even though several theoretical works have dealt with angular redistribution sources, it is still difficult to identify the process inducing the red shift. Moreover, the only existing rocket data capable of giving direct evidence of upward fluxes and of providing a quantitative information about them do not reveal any upward flux. It seems then interesting to focus again on this observed red shift phenomenon. In this aim, for the first time, a transport code is used to try to evaluate the contribution of different redistribution sources to the magnetic-zenith Doppler profile of $\mathrm{H}$ emissions, and especially to its wing on the long wavelength side.

Galand et al. (1997) have described an original way to solve the proton transport equations. The introduction of dissipative forces to describe the energy degradation of protons and $\mathrm{H}$ atoms leads to a form for the proton equation system which remains simple even though angular redistribution is taken into account. Based on this solution, a proton code was developed, and its validation was discussed based on theoretical as well as experimental considerations. As angular redistribution sources can be included in the code, we now propose to use this code in order to investigate the upward fluxes they induce and their possible contribution to the Doppler red shift observed in magneticzenith profiles of $\mathrm{H}$ emissions.

The first angular redistribution source studied is the magnetic mirroring effect. In Sect. 2, the numerical term in the proton transport equation relevant to this effect is first validated. The influence of the magnetic mirror on particle fluxes is thus discussed in depth and its influence on the magnetic-zenith Doppler profile of $H_{\beta}$ emission is evaluated. Section 3 deals with another angular redis- 
tribution source, which is induced through collisions with ambient neutrals. Its influence both on particle fluxes and the $H_{\beta}$ emission profile is the focus of attention. In Sect. 4, a comparison between our model and the existing data which could provide information about the existence of an upward flux is performed: the Proton I rocket experiment and ground-based observations of $\mathrm{H}$ emissions along the magnetic zenith. The possible contribution of the different sources - physical as well as instrumental - which could be at the origin of the red shift of the magnetic-zenith Doppler profile, is reviewed and discussed in the last Sect. 5.

\section{Magnetic mirroring effect}

The magnetic mirroring effect is induced by the convergence of the geomagnetic field lines. It can lead to the upward reflection of downcoming charged particles, here the protons. Conservation of the first adiabatic invariant, the magnetic moment, requires that:

$\frac{\mathrm{d} \mu}{\mathrm{d} s}=-\frac{1-\mu^{2}}{2 \mu B} \frac{\mathrm{d} B}{\mathrm{~d} s}$,

where $\mu$ is the cosine of the pitch angle of the protons defined as the angle between the magnetic field line and the proton velocity. $\mu$ is chosen negative for downward particles and positive for upward particles. The variable $s$ is the spatial coordinate taken along the magnetic field line, positive upward, and $B$ represents the geomagnetic field. Here $B$ is assumed to follow the magnetic field of a centered dipole:

$\frac{1}{B} \frac{\mathrm{d} B}{\mathrm{~d} s}=\frac{-3|\sin \alpha|}{R_{E}+z}$,

where $R_{E}$ is the Earth radius and $z$ the altitude of the studied point in the atmosphere, above sea level. The variable $\alpha$, which depends on the altitude, represents the dip angle, that is the angle between the magnetic field and the horizontal. In the auroral region located at high latitudes, the dip angle varies less than $0.8^{\circ}$ from 80 to $800 \mathrm{~km}$ : the magnetic field lines can be assumed to be straight lines.

\subsection{Validation of the magnetic mirroring term}

Most proton transport models proposed in the literature neglect the magnetic mirroring effect (Jasperse and Basu, 1982; Basu et al., 1990, 1993; Decker et al., 1996). Kozelov (1993) has developed a Monte Carlo code which includes this effect. It was applied to a single constituent atmosphere $\left(\mathrm{N}_{2}\right)$ and for a monoenergetic beam.

In order to validate the numerical term describing the mirroring effect in the proton transport code, we perform a study without collisions. In this case protons do not undergo collisions with neutrals and thus do not suffer energy degradation nor produce $\mathrm{H}$ atoms through capture. The profile in altitude of the proton pitch angle is completely defined by Eq. (1). Several profiles, related to three different initial values of the pitch angle at the highest altitude, are plotted on Fig. 1a in solid lines. These profiles represent the theoretical reference.

When no collision is considered, the proton transport equation [see Eq. (8a) of Galand et al. (1997)] is reduced to:

$\frac{\partial}{\partial s} \Phi_{P}(s, E, \mu)=\frac{1-\mu^{2}}{2 \mu B} \frac{\mathrm{d} B}{\mathrm{~d} s} \frac{\partial}{\partial \mu} \Phi_{P}(s, E, \mu)$,

where $\Phi_{P}$ is the proton flux in $\mathrm{cm}^{-2} \cdot \mathrm{s}^{-1} \cdot \mathrm{eV}^{-1} \cdot \mathrm{sr}^{-1}$ and $E$, the proton energy in $\mathrm{eV}$.

Equation (3) can be solved numerically following the method described in Sect. 3.3 of Galand et al. (1997). Proton fluxes as a function of altitude, energy, and pitch angle can be determined: the pitch angle distributions of proton fluxes are shown in Fig. $1 \mathrm{~b}$ for different altitude levels. What is assumed is the incident downward flux, which is the part of the solid line corresponding to pitch angles between $90^{\circ}$ and $180^{\circ}$ and to an altitude of $800 \mathrm{~km}$. The distributions at lower altitudes and the one corresponding to upward particles at $800 \mathrm{~km}$ are calculated. It has been checked that the shape of the profile is
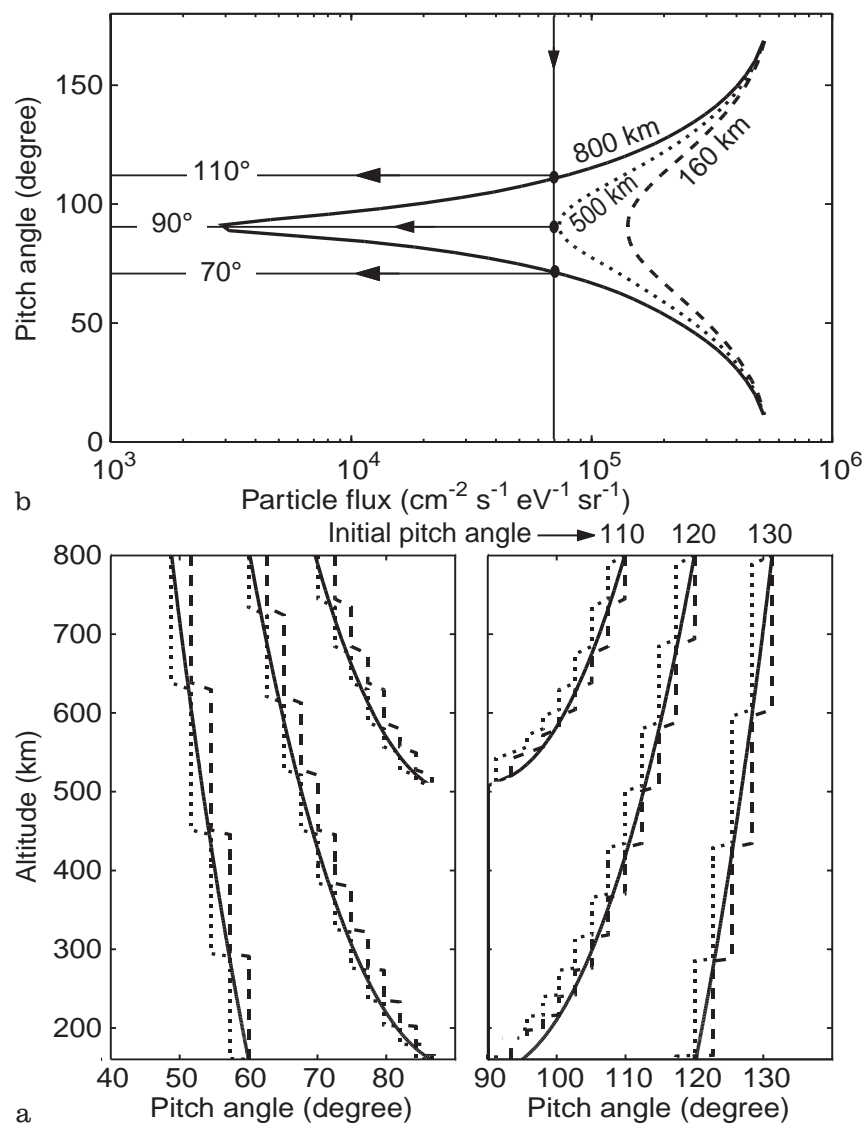

Fig. 1. a Profiles in altitude of the proton pitch angle, for three different initial values, $110^{\circ}, 120^{\circ}$, and $130^{\circ}$ at the top of the atmosphere taken at $800 \mathrm{~km}$. Theoretical profiles are plotted in solid lines and numerical results are shown with dotted and dashed lines. b Proton flux as a function of pitch angle, for different altitudes. The incident flux at $800 \mathrm{~km}$ is represented with a solid line. The proton flux at $500 \mathrm{~km}$ is plotted as a dotted line and the flux at $160 \mathrm{~km}$ with a dashed line. These fluxes have been obtained by neglecting collisions 
independent of proton energy, as it should be for pure magnetic mirroring. It should be emphasized that at $180^{\circ}$, the flux value remains constant with altitude: the magnetic mirror has no effect on particles propagating along the magnetic field line.

Because the assumption of steady state is applied to the proton transport equation [see Sect. 3.1 in Galand et al. (1997)], the proton flux related to a given initial pitch angle at the top of the atmosphere is conserved in altitude as it changes pitch angle according to Eq. (1). Therefore, to determine the altitude profile of the pitch angle of this particle flux, it is enough to follow a constant flux value in altitude on Fig. 1b. For instance, if we choose the initial pitch angle at an altitude of $800 \mathrm{~km}$ (solid line on Fig. 1b) of $110^{\circ}$ the value $\Phi_{0}$ for the proton flux is about $7 \cdot 10^{4} \mathrm{~cm}^{-2} \cdot \mathrm{s}^{-1} \cdot \mathrm{eV}^{-1} \cdot \mathrm{sr}^{-1}$. Then, under the magnetic mirroring effect, the pitch angle of the protons constituting the flux of value $\Phi_{0}$ decreases with the decreasing altitudes: the reflection occurs at an altitude of $500 \mathrm{~km}$ (dotted line on Fig. 1b). Next, protons reverse their path and reach the altitude of $800 \mathrm{~km}$ with an angle of $70^{\circ}$. The magnetic mirroring effect consists of a flux propagation from high to lower pitch angles.

The profiles in altitude of the pitch angles deduced from the proton flux are plotted on Fig. 1a. The solid lines show the theoretical curves, the two lines with steps (dashed and dotted) result from the numerical solution on a discrete pitch angle grid. The relatively good agreement between the reference profiles obtained from Eq. (1) and those deduced from the proton transport equation (3) validates the numerical implementation of the magnetic mirroring term in the proton transport code.

\subsection{Influence on particle fluxes}

For a more realistic case, we study the influence of the magnetic mirroring effect on a proton beam precipitating into a model atmosphere, using the proton code described in Galand et al. (1997). The downward proton flux incident at $800 \mathrm{~km}$ is assumed to be isotropic over the downward hemisphere (Hultqvist, 1979; Basu et al., 1987); its energy distribution is taken equal to a Maxwellian with a characteristic energy of $10 \mathrm{keV}$ and with an energy flux of $1 / \epsilon \mathrm{erg} \cdot \mathrm{cm}^{-2} \cdot \mathrm{s}^{-1}$. The parameter $\epsilon$ is an attenuation parameter which is applied to the incident flux in order to take the spreading of the beam into consideration (Jasperse and Basu, 1982). The atmosphere model is given by MSIS 90 (Hedin, 1991), with a magnetic index $A p$ of 20 and a solar index $F_{10.7}$ of 150 valid for average magnetic and solar conditions. Although the neutral model has an influence on particle fluxes, our conclusions are based on general features of the evolution of these fluxes and are independent of the chosen density distribution. The cross sections of the neutral species $\mathrm{N}_{2}, \mathrm{O}_{2}$, and $\mathrm{O}$ for the ionization, excitation, capture, and stripping reactions are from Basu et al. (1987) and Rees (1989). Moreover the influence of the nonuniformity of the magnetic field on the pitch angle of $\mathrm{H}$ atoms is neglected and no collisional angular redistribution is assumed. Therefore the only source of angular redistribution is, in Sects. 2.2 and 2.3, the magnetic mirroring effect applied to protons. The altitude grid extends from 90 to $800 \mathrm{~km}$ on 200 levels and the energy grid, from 0.1 to $300 \mathrm{keV}$ on 300 levels. The pitch angle grid is uniform in $\mu$, with 20 levels. The downward and upward fluxes obtained by hemispheric integration are presented in Fig. 2. For the display, these fluxes are truncated below $1 \mathrm{~cm}^{-2}$. $\mathrm{s}^{-1} \cdot \mathrm{eV}^{-1}$.

Protons precipitating into the atmosphere lose energy by collisions with ambient neutrals: Fig. 2a illustrates the flux transfer of downward protons from high to lower energies with the decreasing altitudes. Thanks to capture collisions, protons can be neutralized and a downward hydrogen flux is generated, as seen in Fig. 2 b. Above about $300 \mathrm{~km}$, the energy loss is not yet preponderant and the beam just tends to be at the charge equilibrium. At lower altitudes, with a higher and higher atmospheric density, both protons and $\mathrm{H}$ atoms undergo energy degradation. Due to the magnetic mirroring effect, a part of precipitating protons are reflected towards higher altitudes (see Fig. 2c). In the same way as for the downward flux, a part of the upward proton flux can be neutralized through charge-changing reactions. The upward $\mathrm{H}$ atom flux is presented in Fig. 2d. It should be pointed out that the upward beam is mainly composed of $\mathrm{H}$ atoms at low energies, whereas it is protons which dominate at higher energies. This can be explained by the cross section ratio between chargechanging reactions. For instance, at low energies, the capture cross sections related to the proton neutralization are higher than the stripping cross sections associated with the production of a proton from an $\mathrm{H}$ atom.

Figure 2, presenting the different hemispheric-integrated particle fluxes, provides a good survey of the evolution of the proton beam during its propagation throughout the atmosphere. The pitch angle distributions of the fluxes, plotted in Fig. 3 for different altitudes and energies, give another point of view of the evolution of the proton beam and especially illustrate not only the energy degradation, but also the influence of the magnetic mirroring effect. The incident flux, assumed to be isotropic and consisting only of protons, is represented by the solid line corresponding to an altitude of $800 \mathrm{~km}$ and for pitch angles between $90^{\circ}$ and $180^{\circ}$.

At energies greater than the characteristic energy of the incident flux $(10 \mathrm{keV})$, the shape of the flux profiles in pitch angle is illustrated in Fig. 3a. The downward particle fluxes, characterized by a pitch angle greater than $90^{\circ}$, first tend to a charge-exchange equilibrium and then decrease with decreasing altitudes in the dense region of the atmosphere. The decrease is strongest for smaller pitch angles. In a given atmosphere layer, the path of downward particles at low pitch angles is longer than the path of the particles at higher angles: the former particles undergo more collisions and then are degraded faster in energy than the latter. Moreover, owing to the magnetic mirroring effect, there is a flux 

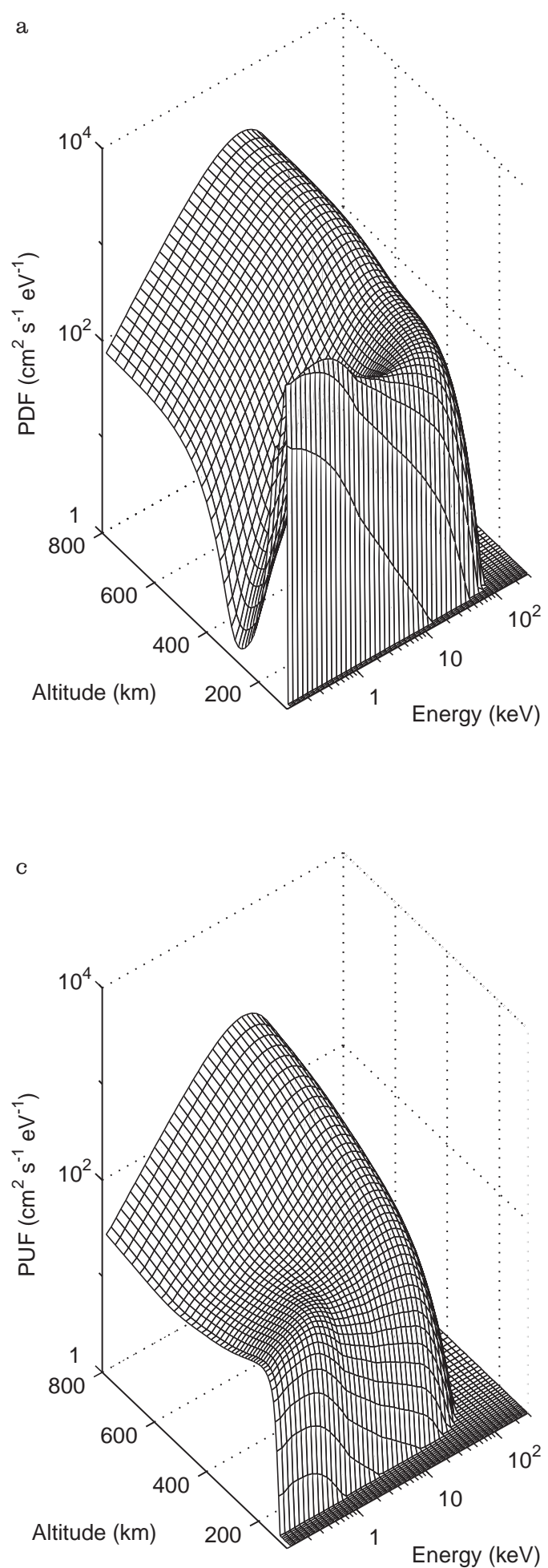

Fig. 2. a Downward proton flux (PDF), b downward $H$ atom flux (HDF), $\mathbf{c}$ upward proton flux (PUF), and $\mathbf{d}$ upward $\mathrm{H}$ atom flux (HUF), as a function of altitude and energy. These fluxes have been

transfer from high to lower pitch angles, with the decreasing altitudes at angles greater than $90^{\circ}$ (downward flux), and with increasing altitudes at angles lower than $90^{\circ}$ (upward flux).

At an energy $E_{1}$, lower than the characteristic energy $E_{0}$ of the incident flux, the profiles in pitch angles are
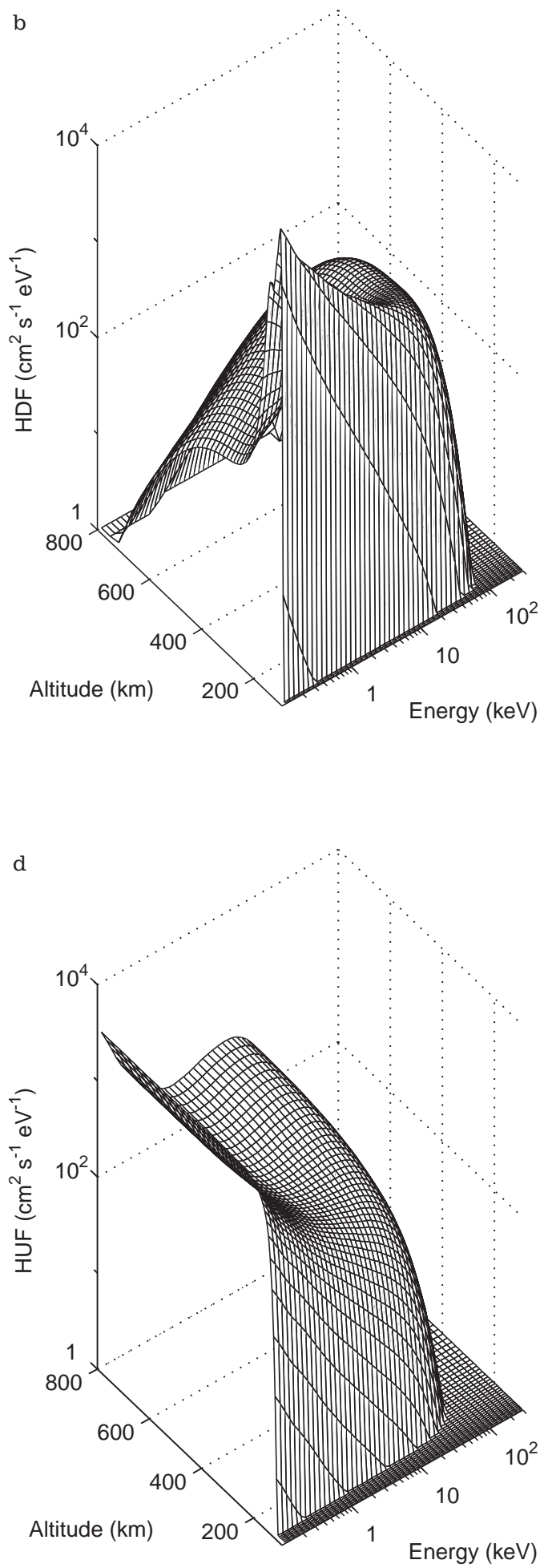

integrated over the upper or lower hemisphere of the pitch angle grid. The upward fluxes result from the magnetic mirror effect

plotted on Fig. 3b. Their shapes are roughly similar to the ones presented in Fig. 3a for higher energies. The main difference lies in the increasing downward flux between $90^{\circ}$ and $110^{\circ}$ at 211 and at $140 \mathrm{~km}$. The decrease in the flux due to flux transfer from $E_{1}$ to lower energies is more than compensated by the gain from 

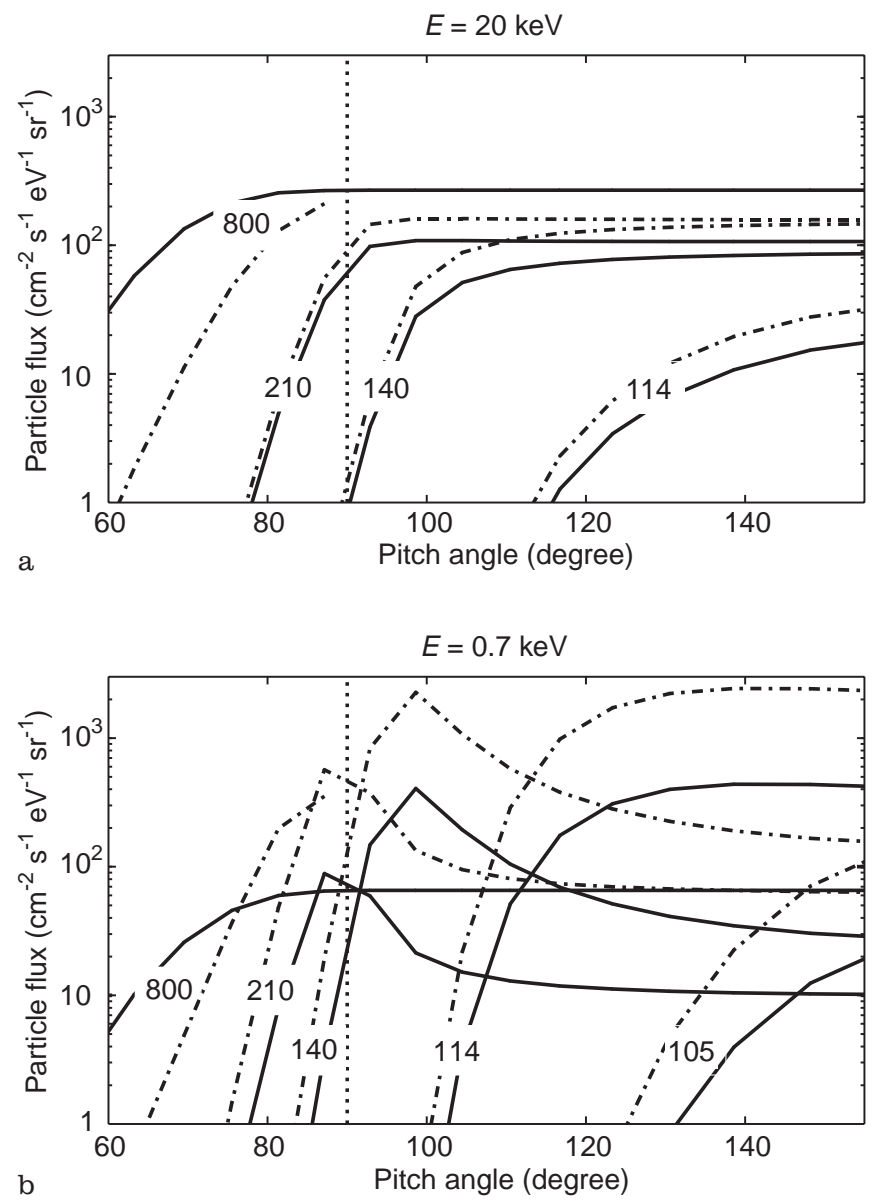

Fig. 3. a Particle fluxes as a function of pitch angle, for different altitude levels (in km) and for an energy of $20 \mathrm{keV}$. Proton flux is shown with solid lines and $\mathrm{H}$ atom flux is plotted with dashed-dotted lines. The upward fluxes, related to pitch angles lower than $90^{\circ}$, result from the magnetic mirror force. b idem, but for an energy of $0.7 \mathrm{keV}$

energies higher than $E_{1}$. Indeed, when $E_{1}$ is lower than $E_{0}$, the gain can exceed the loss. At lower altitudes, the loss is dominant. This is the case in the whole altitude range when the studied energy is greater than $E_{0}$, as shown in Fig. 3a. Below the main energy deposition region - located between 108 and $118 \mathrm{~km}$ for $E_{0}$ of $10 \mathrm{keV}$ - the beam has lost most of its energy, and the fluxes at all energies decrease very quickly.

In both cases illustrated in Fig. 3, the upward fluxes induced by the magnetic mirroring effect begin to be noticeable above around $150 \mathrm{~km}$ and are significant above $200 \mathrm{~km}$. These results are in agreement with those obtained from a Monte Carlo method (Kozelov, 1993). For a monoenergetic incident flux of $10 \mathrm{keV}$ and an isotropic initial pitch angle distribution, noticeable upward fluxes are generated above $200 \mathrm{~km}$. This altitude is a little higher than ours, but the different neutral models, which in Kozelov (1993) was only an $\mathrm{N}_{2}$ atmosphere, can explain this discrepancy.

We should point out that the altitude values given in Fig. 3 depend on the studied energy, on the characteristic energy of the incident flux and on the atmosphere model. Nevertheless, they attest that the magnetic mirroring effect can still act at relatively low altitudes.
At the lowest altitudes, due to the rise of the neutral densities, that is due to the decrease of the mean free path, the effect of magnetic mirror on the proton beam is no longer observable.

\subsection{Influence of magnetic mirroring on the $H_{\beta}$ Doppler profile in the magnetic zenith}

When the magnetic mirroring effect is introduced into the proton transport code, upward fluxes are generated. It is interesting to analyze the influence of this angular redistribution source on the magnetic-zenith Doppler profile of $H_{\beta}$ in order to see if this source alone can explain the red shift observed in measured profiles.

From particle fluxes provided by our model and presented in the previous Sect. 2.2, the emission rate, in photons $\cdot \mathrm{cm}^{-3} \cdot \mathrm{s}^{-1} \cdot \mathrm{eV}^{-1}$, can be deduced:

$\operatorname{Pr}_{1}(s, E, \mu)=2 \pi \sum_{k} \sum_{X=P, H} n_{k}(s) \sigma_{k, X}^{H_{\beta}}(E) \Phi_{X}(s, E, \mu)$,

where $\sigma_{k, X}^{H_{\beta}}$ represents the cross section of the $H_{\beta}$ emission obtained after the excitation of an $\mathrm{H}$ atom by collision between a neutral species $k$ and an energetic particle $X$, a proton $(P)$ or an $\mathrm{H}$ atom $(H)$. If $X$ is a proton, the collision is a capture. The $H_{\beta}$ emission cross sections for $\mathrm{N}_{2}$ and $\mathrm{O}_{2}$ are taken from Van Zyl and Neumann (1980) from $30 \mathrm{eV}$ to $3 \mathrm{keV}$ and from Shen (1993) for higher energies. As far as we know, there is no measurement of the cross sections for collisions with atomic oxygen: the $H_{\beta}$ emission cross sections associated with $O$ have been obtained by dividing the $\mathrm{O}_{2}$ cross sections by a factor of two.

By a change of variables from $(E, \mu)$ to $\left(\lambda, v_{\text {perp }}\right)$, where $\lambda$ is the Doppler-shifted wavelength of the emitted photon $\left(\lambda=\frac{\lambda_{0}}{1-\mu / c \sqrt{2 E / m}}\right)$ and $v_{\text {perp }}$, the velocity of the emitting $\mathrm{H}$ atom perpendicular to the magnetic field $\left(v_{\text {perp }}=\sqrt{1-\mu^{2}} \sqrt{2 E / m}\right)$, the emission rate defined in Eq. (4) becomes:

$$
\begin{aligned}
& \operatorname{PrH}_{2}\left(s, \lambda, v_{\text {perp }}\right) \\
& \quad=\frac{m c}{\lambda_{0}}\left(\frac{\lambda_{0}}{\lambda}\right)^{2} \frac{v_{\text {perp }}}{\sqrt{c^{2}\left(1-\frac{\lambda_{0}}{\lambda}\right)^{2}+v_{\text {perp }}^{2}}} \operatorname{PrH}_{1}(s, E, \mu),
\end{aligned}
$$

with $\mathrm{PrH}_{2}$ in photons $\cdot \mathrm{cm}^{-3} \cdot \mathrm{s}^{-1} \cdot \mathrm{nm}^{-1} \cdot(\mathrm{cm} / \mathrm{s})^{-1}, \lambda_{0}$, the characteristic $H_{\beta}$ wavelength equal to $486.1 \mathrm{~nm}$, and $m$, the mass of an $\mathrm{H}$ atom.

The magnetic-zenith Doppler profile of $H_{\beta}$ can be determined from the following relation:

$D(\lambda)=\iint \mathrm{d} s \mathrm{~d} v_{\text {perp }} \operatorname{Pr}_{2}\left(s, \lambda, v_{\text {perp }}\right)$

where $D$ is in photons $\cdot \mathrm{cm}^{-2} \cdot \mathrm{s}^{-1} \cdot \mathrm{nm}^{-1}$.

The magnetic-zenith Doppler profile of $H_{\beta}$ calculated from the particle fluxes discussed in Sect. 2.2 is presented by the dashed line in Fig. 4. The line-of-sight velocity of the emitting $\mathrm{H}$ atom corresponds to the velocity along the magnetic field line, that is to $\mu \sqrt{2 E / m}$. The negative 


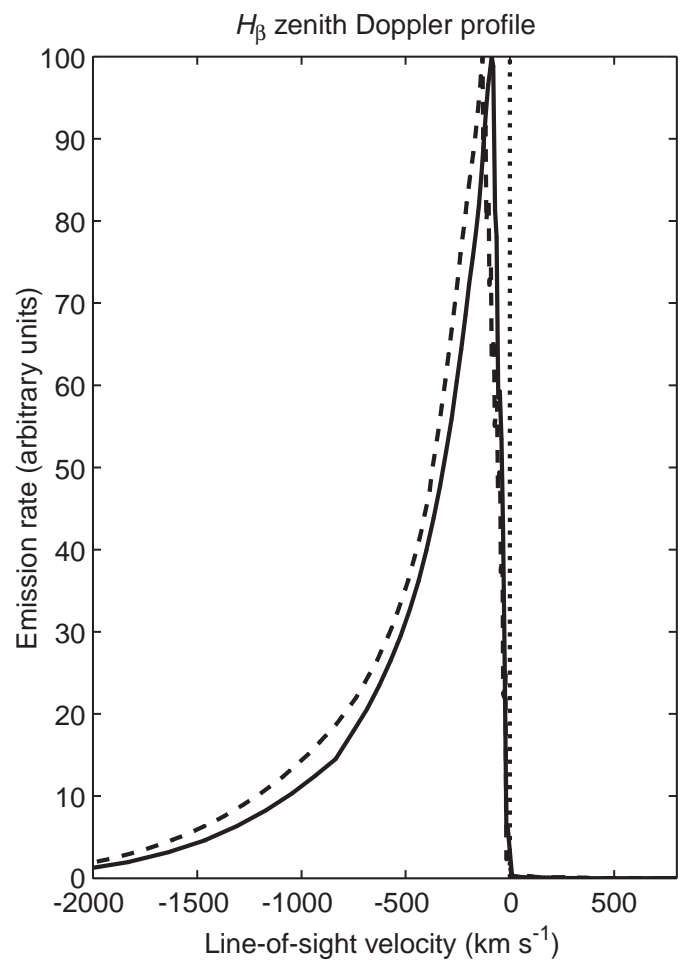

Fig. 4. Normalized $H_{\beta}$ Doppler profiles as a function of line-of-sight velocity, in the magnetic zenith. The dashed line has been computed with a minimum energy of $100 \mathrm{eV}$, the solid line with a minimum energy of $40 \mathrm{eV}$. Both profiles have been normalized individually. These profiles have been obtained by including the magnetic mirror in the model

velocities are relevant to photons emitted by downwardmoving $\mathrm{H}$ atoms and the positive ones to photons emitted by upward-moving $\mathrm{H}$ atoms. As most of the particles are downward, the peak of the profile in Fig. 4 is shifted toward the negative velocities, that is, to wavelengths shorter than $\lambda_{0}$. The peak is blue shifted by $132 \mathrm{~km} \cdot \mathrm{s}^{-1}$, that is about $0.2 \mathrm{~nm}$. However, we should point out that the maximum of the emission is related to the lowest level $E_{\min }$ equal to $100 \mathrm{eV}$, on the particle energy grid. The reason is due to the high values of capture cross sections, mainly of $\mathrm{O}_{2}$ and $\mathrm{O}$, in addition to large emission cross sections at low energies. In the energy deposition region, located between 108 and $118 \mathrm{~km}$ for incident fluxes with $E_{0}$ of $10 \mathrm{keV}$, most of the low-energy protons produced by energy degradation through collisions are neutralized. As a consequence, the $\mathrm{H}$ flux reaches higher and higher values as the energy decreases, and leads to an $\mathrm{H}$-emission maximum at the lowest energy $E_{\min }$.

Faced with this problem, we have reduced the energy $E_{\min }$ until the blue-shifted peak of $H_{\beta}$ Doppler profile becomes independent of $E_{\min }$ : a minimum energy of $40 \mathrm{eV}$ is required. Using such a low energy required extrapolation of the collision cross sections, and also requires examination of the validity of the assumption of a continuous energy degradation. For protons, the main reaction below $1 \mathrm{keV}$ is the capture with an energy loss lower than $4 \mathrm{eV}$, but for $\mathrm{H}$ atoms the energy loss is at least $17 \mathrm{eV}$. Nevertheless, it has to be pointed out that the particle fluxes presented in Sect. 2.2 are not affected by the reduction of $E_{\min }$, since the energy transfer occurs from high to lower energy. Moreover, the profile obtained with $E_{\min }$ equal to $40 \mathrm{eV}$ and plotted as a solid line in Fig. 4 is very close to the one obtained with $E_{\min }$ equal to $100 \mathrm{eV}$. The discrepancy amounts to a difference between the two profiles less than $44 \mathrm{~km} \cdot \mathrm{s}^{-1}$, that is about $0.07 \mathrm{~nm}$. Therefore it seems reasonable to state that the theoretical profile for the given atmosphere model, cross section set, and input flux is in the range of those two profiles. It has to be pointed out that if those two profiles have the same normalization, they coincide for wavelengths related to energies greater than $100 \mathrm{eV}$, as the particle fluxes are the same in this energy range. At wavelengths corresponding to lower energies, the Doppler profile obtained with a minimum energy of $40 \mathrm{eV}$ has an higher intensity - taking into account the emission of more particles - compared with the profile related to a minimum energy of $100 \mathrm{eV}$.

The spreading of the Doppler profile toward the positive line-of-sight velocity, that is, toward wavelengths longer than $\lambda_{0}$, is negligible: the contribution of the upward particles redistributed by magnetic mirroring effect is insignificant on the $H_{\beta}$ emission profile. As pointed out in Sect. 2.2, the upward flux is generated in the less dense regions, largely above the energy deposition region, located here around $113 \mathrm{~km}$, where the blue-shifted emission induced by downward particles is produced. Therefore, for the given incident flux the magnetic mirroring effect alone cannot generate a significant red shift in the magnetic-zenith profile. The same conclusion is valid for $H_{\alpha}$ emission whose related cross sections can be obtained from Van Zyl and Neumann (1980) and Yousif et al. (1986). Moreover, variation of the characteristic energy $E_{0}$ of the incident flux has very little effect on the red shift. The blueshifted peak also remains nearly unchanged as the maximum of the emission is induced by low-energy particles. Only the violet wing of the profile, the spreading of the profile from the peak towards the larger downward velocities, increases in intensity and extends to more negative velocities with an increase of $E_{0}$ : the contribution of very energetic particles to the emission profiles is more important. For a decrease of $E_{0}$, the violet wing is reduced, owing to a reduction of high-energy particles. The variation of the energy flux, $Q_{0}$, of the incident proton beam induces a proportional variation in the particle number flux, so the normalized profile presented in Fig. 4 remains unchanged. Thus, it appears that the magnetic mirroring effect, applied to an isotropic downward incident proton flux, generates an upward flux which is not sufficient to induce a significant physical red shift in the magnetic-zenith Doppler profiles of $\mathrm{H}$ emissions. This result confirms the early work of Bagariatskii (1958a, b).

\section{Collisional angular redistribution}

We now evaluate the contribution of another angular redistribution source: collisional angular redistribution. 
The magnetic mirror force is neglected in this section. Up to now, the different reactions considered between neutral species and energetic hydrogen particles were ionization, excitation, and charge-changing reactions. We now drop the forward-scattering approximation and take angular scattering into account.

The aim of this section is to discuss the influence of collisional angular redistribution on particle flux and its contribution to the red shift of the magnetic-zenith Doppler profile of $\mathrm{H}$ emissions. However, such a study is restricted due to the lack of knowledge about some of the input parameters. The cross sections of the elastic scattering as well as the phase functions specifying the angular redistribution are not all available for the three neutral species considered here, that is for $\mathrm{N}_{2}, \mathrm{O}_{2}$, and $\mathrm{O}$. Rather than assuming cross sections for $\mathrm{O}_{2}$ and $\mathrm{O}$ we limit our study to a single constituent atmosphere. Its density profile in altitude is obtained from the model used in Sect. 2.2, derived from MSIS 90, and converted to $100 \%$ nitrogen: only nitrogen is considered and its density is taken equal to the sum of the different neutral densities provided by MSIS 90. The cross sections for elastic scattering are taken from Kozelov and Ivanov (1992). Cross sections for the other reactions are similar to the ones used in Sect. 2.2, that is from Basu et al. (1987) and Rees (1989).

Measured differential cross sections (Fleischmann et al., 1967, 1974; Cisneros et al., 1976; Van Zyl et al., 1978; Newman et al., 1986; Johnson et al., 1988; Gao et al., 1990), show that protons and $\mathrm{H}$ atoms undergo angular redistribution during collisions both of charge conservation and of charge changing. Therefore, angular redistribution is considered not only during elastic scattering, but also during capture and stripping reactions. As for ionization or excitation, the forward-scattering approximation is assumed. These same measurements show that the phase function depends on the energy of the incident particle, and the importance of the angular scattering increases with decreasing energies. Nevertheless, owing to the sparse information about that energy dependence, we simplify the energy dependence of phase function. Because the angular redistribution is very weak above $1 \mathrm{keV}$, the forwardscattering approximation is assumed (Basu et al., 1993). We thus apply angular redistribution only below $1 \mathrm{keV}$.

The phase function is taken equal to the Rutherford formula used for electrons (e.g., Stamnes, 1980): it is centered on the incident pitch angle before collision, and the screening parameter, which controls the peaked characteristic of the phase function, is taken equal to 0.001 . This value has been estimated from the few differential cross section data which are available to date (Fleischmann et al., 1967, 1974; Cisneros et al., 1976; Van Zyl et al., 1978; Newman et al., 1986; Johnson et al., 1988; Gao et al., 1990).

At last, the conservation of particle number in Eq. (8) in Galand et al. (1997) requires that the phase function $\zeta$ is normalized over the pitch angles before the collision, that is: $\int_{-1}^{1} \zeta\left(\mu_{i} \rightarrow \mu_{f}\right) \mathrm{d} \mu_{i}=1$,

with $\mu_{i}$ and $\mu_{f}$, the cosines of the pitch angle of the energetic particle before and after the collision, respectively. The integral over the pitch angles is performed on the given angle grid.

\subsection{Influence on particle fluxes}

In order to determine the particle flux, we solve the transport equations as described in Galand et al. (1997). The introduction of collisional angular redistribution in those equations does not seriously complicate the solution, as described in Sects. 3.2 and 3.3 in Galand et al. (1997). Unlike the case of the magnetic mirroring effect, the upward flux generated by collisional angular redistribution affects the downward flux. Therefore, more than one downward and upward calculation cycle in the simulated atmosphere is needed. Typically, convergence of the results is obtained in less than 15 iterations.

The incident flux at the top of the atmosphere is the same as that chosen in Sect. 2.2: this downward flux is isotropic and its energy distribution is a Maxwellian with a characteristic energy, $E_{0}$, of $10 \mathrm{keV}$ and with an energy flux, $Q_{0}$, of $1 / \epsilon \mathrm{erg} \cdot \mathrm{cm}^{-2} \cdot \mathrm{s}^{-1}$. The altitude, energy, and angle grids are also similar to those used in Sect. 2.2, except for an energy grid of 200 levels. The downward and upward fluxes obtained in the case of collisional angular redistribution are plotted in Fig. 5, in the same way as the fluxes presented in Fig. 2 described in Sect. 2.2. As for that previous study for the magnetic mirroring effect, both the neutralization of part of the incident proton beam due to capture, and the energy transfer from high to low energy are observed in the downward flux presented in Fig. 5a, b. As the angular redistribution during a collision is assumed to occur only below $1 \mathrm{keV}$, the upward flux is generated only below this energy. Unlike the magnetic mirroring effect, the collision process directly generates both proton and $\mathrm{H}$ atom upward fluxes and acts at low altitudes: the upward flux is produced where collisions are numerous on the proton beam, that is, in the energy deposition region located around $113 \mathrm{~km}$ for an $E_{0}$ of $10 \mathrm{keV}$. Above $103 \mathrm{~km}$, the upward flux begins to be significant. Above the energy deposition region, the upward flux acts as a source for the downward flux. As a result, the decrease observed in Fig. $2 a, b$ around $200 \mathrm{~km}$ on the downward flux is largely attenuated in Fig. 5.

A study of particle flux as a function of the pitch angle provides more details about these general features. At high energies (above $1 \mathrm{keV}$ ) no angular scattering occurs, and the flux undergoes only energy degradation through collisions and decreases with decreasing altitude, as illustrated in Fig. 6a. This decrease is more important at pitch angles near $90^{\circ}$, at which the particle path is longer in a given altitude layer. The downward 

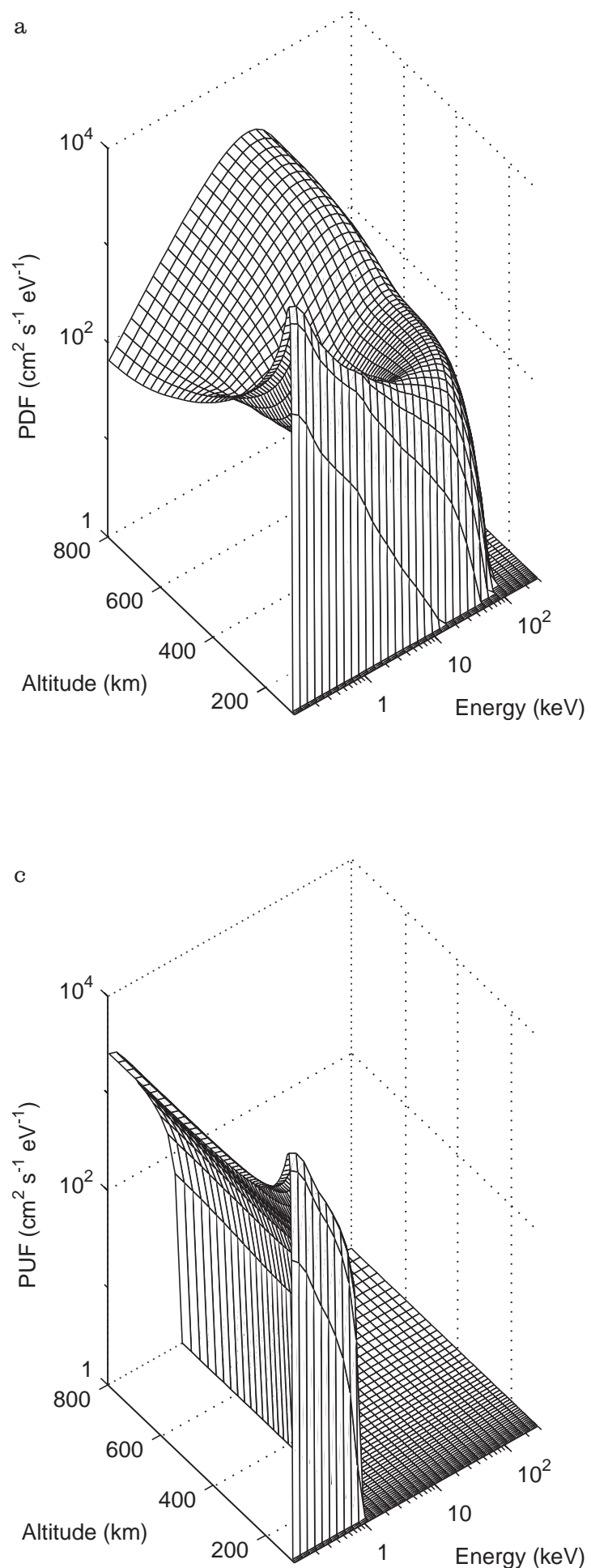
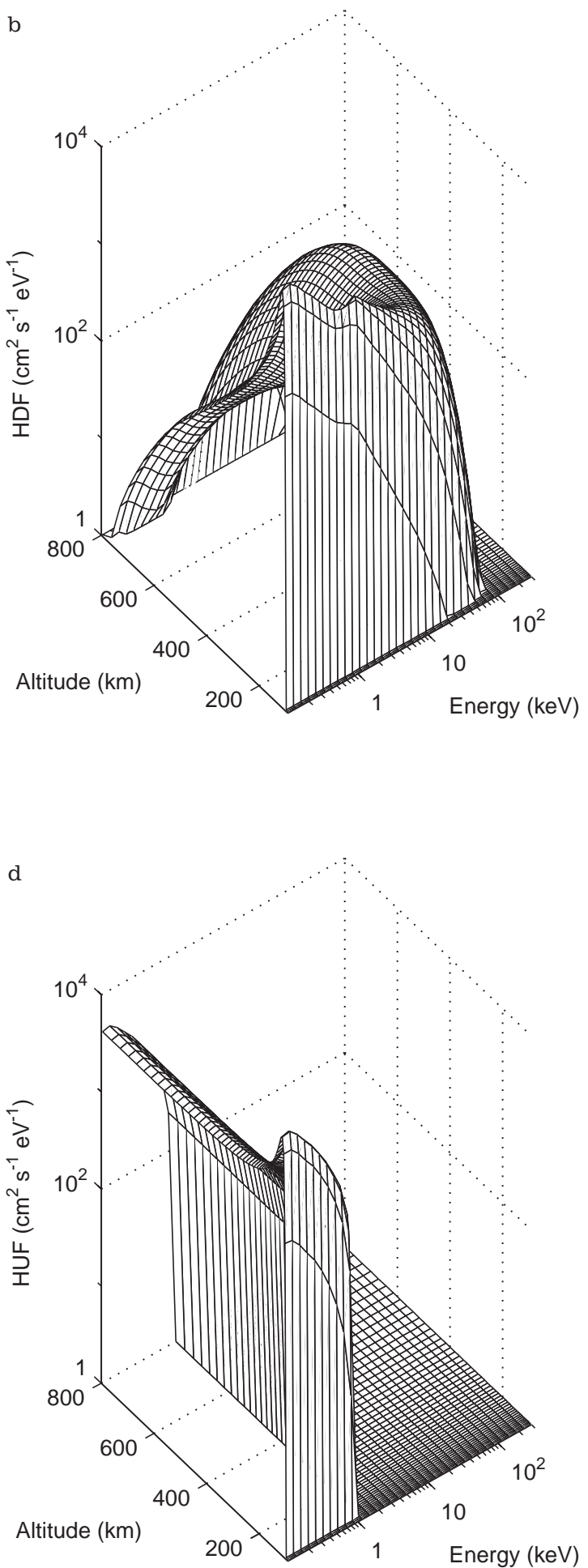

Fig. 5. a Downward proton flux, $\mathbf{b}$ downward $\mathrm{H}$ atom flux, $\mathbf{c}$ upward proton flux, and $\mathbf{d}$ upward $\mathrm{H}$ atom flux, as a function of altitude and energy. These fluxes have been integrated over the half pitch angle grid. The upward fluxes result from the effect of collisions

flux in this case is similar to the one obtained in the magnetic mirror case (see Fig. 3a).

Below $1 \mathrm{keV}$, the particles undergo angular redistribution in addition to the energy transfer from higher energies. An upward flux is generated at low altitudes in the dense atmosphere and is already important at $105 \mathrm{~km}$ as shown in Fig. 6b. Multiple scattering renders the flux more isotropic in pitch angle even above the energy deposition region. At high altitudes, with a less dense atmosphere, the changes in energy and pitch angle are less important, which leads to a decrease in the upward flux.

Because the upward flux is generated at low altitude where the atmosphere is dense, we expect a more 

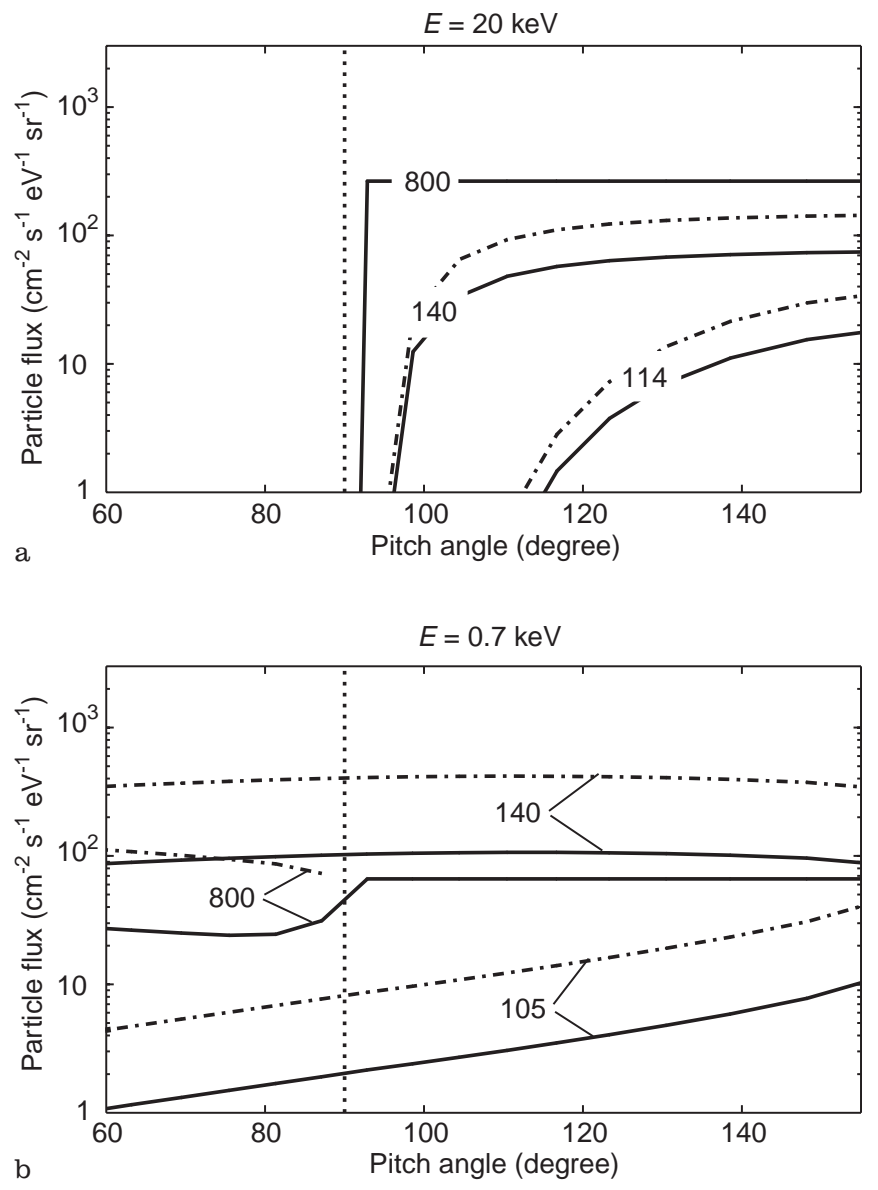

Fig. 6. a Particle fluxes as a function of pitch angle, for different altitude levels (in $\mathrm{Km}$ ) and for an energy of $20 \mathrm{keV}$. Proton flux is shown with solid lines and $\mathrm{H}$ atom flux is plotted with dashed-dotted lines. b idem, but for an energy of $0.7 \mathrm{keV}$. The upward fluxes related to pitch angles lower than $90^{\circ}$ - result from collisions

significant red shift in the magnetic-zenith Doppler profile on $\mathrm{H}$ emissions, compared to the case of the magnetic mirroring effect.

\subsection{Influence of collisional angular redistribution on the $H_{\beta}$ Doppler profile in the magnetic zenith}

The $H_{\beta}$ Doppler profile viewed in the magnetic zenith and deduced from the particle fluxes presented in Sect. 3.1 is calculated in the same way as in Sect. 2.3. The result is presented in Fig. 7. Its shape is in agreement with expectations: a blue-shifted peak due to the downward major flux and a red shift due to the upward flux generated by collisions.

Unlike the case of the magnetic mirror, the blueshifted peak is here relatively wide. When they penetrate at low altitudes, the particles undergo a variation of their pitch angle and those whose angle becomes close to $90^{\circ}$ are quickly absorbed in the dense atmosphere. Therefore near $100 \mathrm{eV}$ the particle flux does not increase so abruptly compared to the case without collisional angular redistribution (see Fig. 2b and d). This relatively

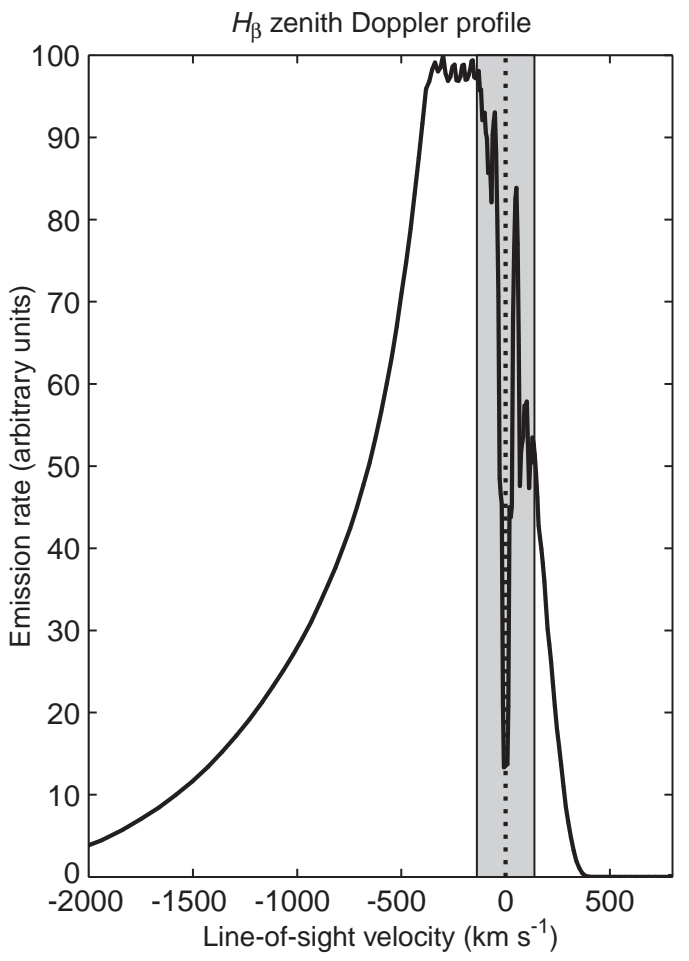

Fig. 7. Normalized $H_{\beta}$ Doppler profiles as a function of line-of-sight velocity, in the magnetic zenith. The shaded area represents the part of the profile which can be affected by particles of energy lower than the minimum energy of the computation grid, that is $100 \mathrm{eV}$. This profile has been obtained from the model including collisional angular redistribution

constant flux at low energies, and the large $H_{\beta}$ cross sections in this energy range, lead to a broad peak in the Doppler profile.

The shaded area extends from minus 150 to plus $150 \mathrm{~km} \cdot \mathrm{s}^{-1}$, i.e., $\pm 0.2 \mathrm{~nm}$ around $\lambda_{0}$. It represents the part of the profile which could be affected by particles of energy below the lowest level on the energy grid, that is $100 \mathrm{eV}$. The profile in Fig. 7 is therefore an underestimate in this area, as it results only from high-energy $\mathrm{H}$ atoms with pitch angles near $90^{\circ}$. In future work, we will need to extend the computation to lower energies to determine the proper profile around $0 \mathrm{~km} \cdot \mathrm{s}^{-1}$. The continuous slowing down approximation we applied in our model will be still valid below $100 \mathrm{eV}$ : the relative energy loss of elastic scattering, the major reaction in this energy range, has a very low value, less than few per cent [see Sect. 2 in Galand et al. (1997)]. But such a computation requires elastic cross section data at low energies which are not available to date, as far as we know. Nevertheless, if we assume that the emission rate in the shaded area will remain lower than the one at the blue-shifted peak, the part of the normalized profile in the nonshaded area will not be significantly affected by an extension of the energy grid towards lower energies.

For the chosen phase function, the profile exhibits a significant red-shifted contribution. The long-wavelength wing - positive-line-of-sight velocity wing extends up to almost $400 \mathrm{~km} \cdot \mathrm{s}^{-1}$, that is about 
$0.6 \mathrm{~nm}$ or $0.84 \mathrm{keV}$. This value is related to $1 \mathrm{keV}$, the highest energy for which the angular redistribution during a collision is applied. The limiting energy for the long-wavelength wing is a little lower than $1 \mathrm{keV}$ : the particles need to undergo many collisions, and thus a certain amount of energy loss, before being significantly redistributed in angle. As expected, the upward flux generated in the dense atmosphere has an influence much more important on the Doppler profile than the upward flux induced at higher altitudes by magnetic mirroring. We will discuss the conditions under which this physical red shift can be observed in Sect. 4 .

The influence of $E_{0}$ and $Q_{0}$, parameters of the incident flux, on the emission profile is the same as discussed in Sect. 2.3 and the red shift is not affected, because it is due to low-energy particles. A similar profile is obtained for $H_{\alpha}$. When angular scattering is extended to energies higher than $1 \mathrm{keV}$, the red shift will extend to a higher line-of-sight velocity, that is to longer wavelengths. Finally, if the phase function $\zeta$ is taken more peaked, the red extent of the profile is reduced.

\section{Comparison with observations}

The most valuable data which could confirm an upward flux and the origin of the red shift, would be direct proton and $\mathrm{H}$ atom flux measurements acquired aboard a rocket over a range of altitudes, energies, and pitch angles. Such data would show if energetic particles are significantly redistributed in pitch angle and, if so, in which atmospheric region the upward flux is generated. As this study (Sects. 2 and 3) shows, upward flux at low altitudes, below $150 \mathrm{~km}$, originates from collisions; on the other hand, a high-altitude upward flux favors spreading or magnetic mirroring as the source. As far as we know, the only rocket observations providing angular data are from the Proton I experiment (Söraas et al., 1974, 1994).

During that rocket flight, pitch angle distributions of proton and $\mathrm{H}$ atom flux were acquired at high energies, above $20 \mathrm{keV}$, and for different altitudes below $145 \mathrm{~km}$ (Söraas et al., 1994). A successful comparison of the energy distribution between the Proton I data and our proton transport code neglecting angular scattering served to validate our transport calculation (Galand et al., 1997). The data are, however, not suitable to separate sources of angular redistribution of precipitating protons.

The Proton I data show no significant upward flux and the computation results, obtained neglecting angular redistribution in the model, fit the data quite well. The theoretical studies concerning magnetic mirror (see Sect. 2) and collisional angular redistribution (see Sect. 3) offer an explanation for this absence of upward flux in the Proton I data. The pitch angle distributions have been acquired below $150 \mathrm{~km}$, below the region where the magnetic mirroring is expected to have significant influence, as discussed in Sect. 2.2. The angular redistribution due to collisions is generated in this altitude region, but at low energies, mainly below
$1 \mathrm{keV}$, an energy range where no useable data from Proton I experiment are available. Therefore no information about a possible upward flux and about the red shift observed on Doppler profiles of $\mathrm{H}$ emissions viewed along the magnetic zenith can be deduced from these rocket data.

Coordinated measurements between a ground-based spectrometer measuring the $\mathrm{H}$ Doppler profile in the magnetic zenith and a satellite or rocket flying over the observed region of the auroral atmosphere and giving information about the incident proton flux would also shed light on the importance of angular scattering. In the absence of such coordinated observations, we are limited to qualitative comparisons with data.

We have calculated $H_{\beta}$ Doppler profiles from particle fluxes as explained in Sect. 2.3. However, no angular redistribution is considered here. The atmosphere model and cross section sets, as well as the different computation grids are those described in Sect. 2.2. The incident flux is isotropic over the downward hemisphere and its energy distribution is Maxwellian with an energy flux $Q_{0}$ of $1 / \epsilon \mathrm{erg} \cdot \mathrm{cm}^{-2} \cdot \mathrm{s}^{-1}$. We have performed the calculation for an incident flux of characteristic energy of $10 \mathrm{keV}$ and $1 \mathrm{keV}$. The theoretical $H_{\beta}$ Doppler profiles in the magnetic zenith are plotted as dashed lines on Fig. 8.

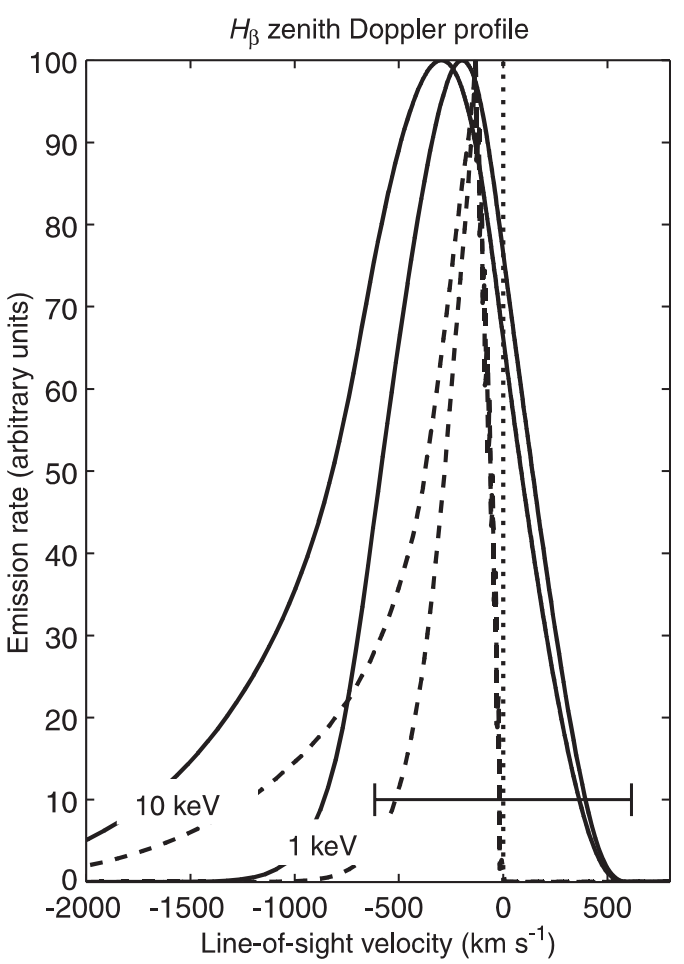

Fig. 8. Normalized $H_{\beta}$ Doppler profiles as a function of line-of-sight velocity, in the magnetic zenith, for two different characteristic energies $E_{0}$ of the incident proton flux. The profiles plotted with dashed lines represent the profiles obtained before convolution. The profiles shown with solid lines have been obtained after convolution with the instrumental function. All profiles have been computed with a model neglecting all angular redistribution. The resolution taken for the instrument is $1 \mathrm{~nm}$ : the associated spreading is shown with a bar around $0 \mathrm{~km} \cdot \mathrm{s}^{-1}$ above the line-of-sight velocity axis 
Because all sources of angular redistribution are neglected in this section, the profiles exhibit no red shift. The violet wing - that part of the profile from the peak to higher downward line-of-sight velocities - depends on $E_{0}$. For high $E_{0}$, it is more intense due to the increased contribution of high-energy particles. However, the blue shift of the peak remains unchanged because the maximum of the emission is due to low-energy particles emitting in the dense lower atmosphere.

In order to compare the theoretical profiles with the observed ones, it is necessary to convolve the former with the instrumental function $F$. This function is assumed to be triangular, as representative of spectrometers with a half width equal to the resolution of the typical instruments used to measure the experimental profiles (see Fig. 9), that is to $1 \mathrm{~nm}$. The profiles obtained after convolution are presented as solid lines in Fig. 8. Due to different violet wings of the profiles before convolution, the maximum of the emission occurs at a wavelength (line-of-sight velocity) which depends on the characteristic energy $E_{0}$ of the incident flux. Moreover, the instrumental resolution is not enough to prevent a significant influence of the blueshifted peak on the part of the profile related to a positive line-of-sight velocity: an apparent red shift of instrumental origin is generated. As an illustration, the spreading width of the instrumental function, $2 \mathrm{~nm}$, is represented as a horizontal bar in Fig. 8. At a velocity of 0 , that is at the characteristic wavelength $\lambda_{0}$, the convolution of the Doppler profile with the instrumental function takes into account points from minus 615 to plus $615 \mathrm{~km} \cdot \mathrm{s}^{-1}$.

Similar profiles are obtained for $H_{\alpha}$. However, it has to be stressed that the convolved $H_{\alpha}$ profile is more peaked than that of $H_{\beta}$, when represented as a function of line-of-sight velocity. The reason is the different characteristic wavelengths of the two Balmer lines, longer for $H_{\alpha}$ than for $H_{\beta}$. The same wavelength resolution leads to less spreading in velocity in the case of $H_{\alpha}$. Finally, it has to be pointed out that the minimum energy of the computation grid has an influence on the shift of the peak, as discussed in Sect. 2.3. As previously stated, a minimum energy of $40 \mathrm{eV}$ solves this problem. The discrepancy between the profiles obtained - before or after convolution - with $E_{\min }$ equal to $40 \mathrm{eV}$ and with $E_{\min }$ equal to $100 \mathrm{eV}$ is less than $40 \mathrm{~km} \cdot \mathrm{s}^{-1}$, that is less than $0.067 \mathrm{~nm}$.

Figure 9 shows the experimental profiles provided by Eather (1967) and valid for a resolution of $1 \mathrm{~nm}$. The previous study can help to shed some light on this set of data. For example, the relatively large discrepancies between the violet wings of the different measured profiles can be explained by incident proton fluxes, at the origin of the H-emission profiles, of different $E_{0}$, and more generally of different energy distributions. The theoretical profile obtained for $E_{0}$ equal to $10 \mathrm{keV}$ is added as the thick solid line on Fig. 9. It fits quite well with the data set, not only for the blue-shifted peak but also for the red shift, although this profile has been obtained neglecting all angular redistribution sources. Therefore it appears that the red shift observed in the measurements can be explained by the resolution of the instruments. To go further in our investigations concerning the origin of the red shift, we need experimental profiles of a much better resolution. Recently Sigernes (1996) provided a magnetic-zenith profile of $H_{\alpha}$ acquired with a half-meter Ebert-Fastie spectrometer with a resolution of $0.25 \mathrm{~nm}$. Sigernes (1996) interpreted the red shift as due to instrumental broadening. However, no direct comparison between a model and the magnetic-zenith Doppler profile has been performed as yet.

In Sect. 3.2, a significant physical red shift was obtained through collisional angular redistribution, for a given phase function. The profile extends up to $0.6 \mathrm{~nm}$ on the long-wavelength side. In order to observe this red shift, a resolution of $0.2 \mathrm{~nm}$ would be necessary. In this case, two-thirds of the physical red shift is not affected by the blue wing despite the spreading due to the instrument. Thus, the instrumental red shift cannot extend more than $0.2 \mathrm{~nm}$, which is more or less the upper limit of the shaded area in Fig. 7.

\section{Discussion}

One of the goals of this paper is to try to shed some light on measurements which could provide information about the possible angular redistribution of auroral precipitating protons. In order to accomplish these

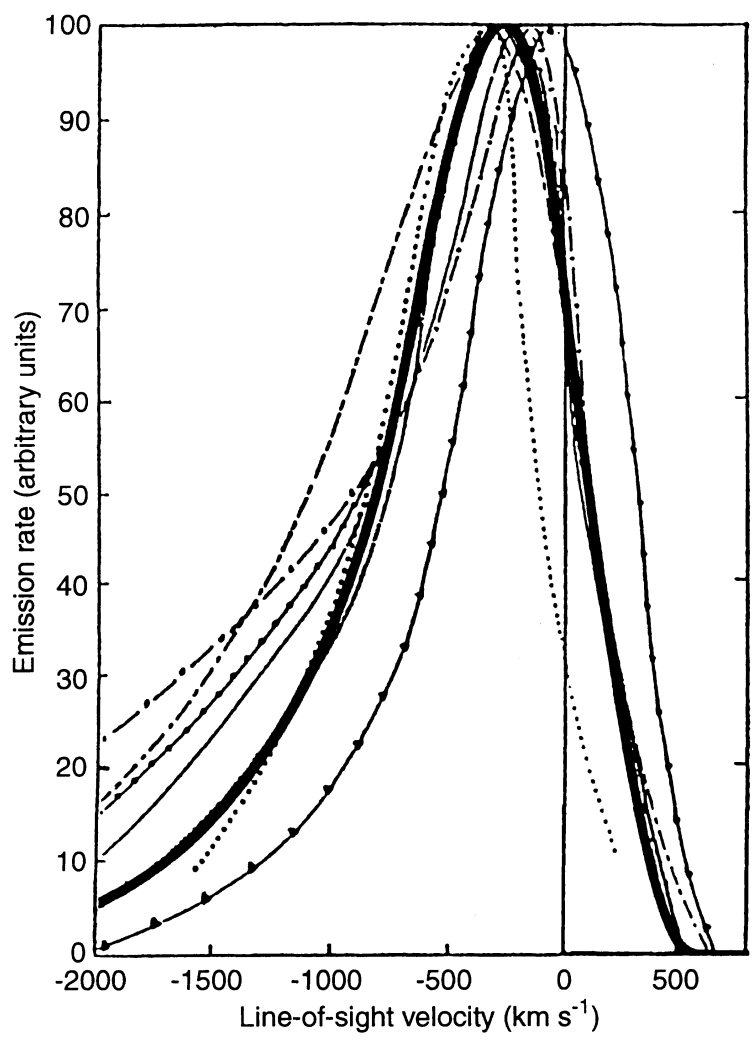

Fig. 9. Experimental measurements of auroral hydrogen line profiles in the magnetic zenith, as a function of line-of-sight velocity (Eather, 1967). The theoretical profile obtained with $E_{0}$ equal to $10 \mathrm{keV}$ is added in thick solid line 
goals, we have undertaken two theoretical studies using our proton transport code which can include two different angular redistribution sources.

Section 2 deals with the magnetic mirroring effect applied to protons and induced by the convergence of the geomagnetic field lines. The numerical term taking into account this process in the proton transport equation was validated in a theoretical study without collisions. It induced a proton flux transfer from high to low pitch angles, which generates an upward flux. By including collisions in the model, an $\mathrm{H}$ atom flux was obtained through neutralization of the reflected protons. The magnetic mirror was found to act mainly above about $200 \mathrm{~km}$, in a region where the mean free path of the protons is long. However, the upward flux produced by this physical process was insufficient to induce a significant red shift on the H Doppler profile. This result confirms the early work of Bagariatskii (1958a, b). We have found that neglecting the effect of the magnetic mirror is valid for any incident proton flux whose angle distribution is isotropic, as most commonly observed.

In Sect. 3, we turned our attention to another angular redistribution source, the one acting through collisions. Due to the lack of quantitative information about several of the relevant input parameters, this study was restricted to an $\mathrm{N}_{2}$ atmosphere, and the phase function was assumed constant and was applied only below $1 \mathrm{keV}$. Unlike the magnetic mirror, the angular redistribution was found to act mainly at low altitudes, near the peak of the energy deposition. Therefore, the upward flux generated in a dense atmosphere can induce a much more intense red shift than that of the magnetic mirror. The extension of the red wing of the profile was found to be related to the energy below which we assumed the angular redistribution. The intensity of the red shift depends on the phase function chosen. However, despite the uncertainties in the phase function, it appears that the collisions could induce a significant and observable physical red shift.

These two theoretical studies are consistent with the Proton I particle flux measurements. The absence of significant upward flux in those data was explained by too high an energy range, where the collisional angular redistribution does not act, and by too low an altitude range to observe the effect of the magnetic mirror.

We have also performed a comparison of our model with a set of $\mathrm{H}$ Doppler profiles viewed in the magnetic zenith. It has been shown that the resolution of the instruments used for these data has been insufficient, so that the blue shift peak contributes to the extension of the profile toward the long-wavelength side: the observed red shift is mainly induced by the instrument, which can hide any upward particle contribution. Therefore, the data to which we have access cannot provide information about upward fluxes inside the proton beam. This emphasizes the need for better data and the importance of theoretical models to aid in their interpretation.

To improve the modeling of proton aurora, especially of upward fluxes and the existence of a possible physical red shift on the H Doppler profile, we need more data concerning several input parameters dealing with the collisional angular redistribution: elastic cross sections, cross sections at low energies, and phase functions. Other angular redistribution sources must also be investigated. In the present study, the results are assumed to be valid in the center of the proton beam and the spreading due to the path of the neutral $\mathrm{H}$ atoms is taken into consideration through an attenuation parameter, denoted $\epsilon$. The variation of the $\mathrm{H}$ atom pitch angle, due to its path across converging magnetic field lines, is neglected. Polar orbiting satellite data have shown that the $90^{\circ} \mathrm{H}$ atom population can become predominant at the equatorward edge of the proton aurora (Sharber, 1981). Kozelov (1993) and more recently Jasperse (1997) have investigated the spreading of the proton beam using a Monte Carlo method and a theoretical approach solving the transport equations, respectively. Their works confirm the importance of this process, which predominantly acts above about $150 \mathrm{~km}$, therefore above the energy deposition region where the $\mathrm{H}$ excitation is maximum. Finally, along with theoretical investigations, rocket measurements mainly at the lowenergy range would provide crucial information about collisional angular redistribution. Ground-based observations of $\mathrm{H}$ emission profiles in the magnetic zenith with better resolution than the profiles analyzed here would be of a great interest, too. As discussed in Sect. 4, an instrument passband $\leq 0.2 \mathrm{~nm}$ is needed to identify a physical red shift induced by collisions.

Acknowledgements. We would like to thank A. Richmond for really enriching discussions and helpful comments, as well as M. H. Rees for his valuable support. We are indebted to F. Söraas for useful comments on Proton I data. M. G. gratefully acknowledges the financial support of the Advanced Study Program and the High Altitude Observatory, divisions of the National Center for Atmospheric Research (NCAR). - NCAR is sponsored by the National Science Foundation.

The Editor-in-Chief thanks C. S. Deehr and another referee for their help in evaluating this paper.

\section{References}

Bagariatskii, B. A., The influence of proton spiral trajectories on the Doppler profile of auroral hydrogen lines, Sov. Astron. AJ, 2, 87-96, 1958a.

Bagariatskii, B. A., The zenith-angle distribution function of auroral protons, Sov. Astron. AJ, 2, 453-453, 1958 b.

Basu, B., J. R. Jasperse, R. M. Robinson, R. R. Vondrak, and D. S. Evans, Linear transport theory of auroral proton precipitation: a comparison with observations, J. Geophys. Res., 92, 59205932, 1987.

Basu, B., J. R. Jasperse, and N. J. Grossbard, A numerical solution of the coupled proton-H atom transport equations for the proton aurora, J. Geophys. Res., 95, 19069-19078, 1990.

Basu, B., J. R. Jasperse, D. J. Strickland, and R. E. Daniell, Transport-theoretic model for the electron-proton-hydrogen atom aurora, 1. Theory, J. Geophys. Res., 98, 21517-21532, 1993.

Cisneros, C., I. Alvarez, C. F. Barnett, and J. A. Ray, Differential scattering cross sections of hydrogen and deuterium atoms in nitrogen, Phys. Rev. A, 14, 84-87, 1976.

Decker, D. T., B. V. Kozelov, B. Basu, J. R. Jasperse, and V. E. Ivanov, Collisional degradation of the proton-H atom fluxes in 
the atmosphere: a comparison of theoretical techniques, $J$. Geophys. Res., 101, 26947-26960, 1996.

Eather, R. H., Red shift of auroral hydrogen profiles, J. Geophys. Res., 71, 5027-5032, 1966.

Eather, R. H., Auroral proton precipitation and hydrogen emissions, Rev. Geophys., 5, 207-285, 1967.

Fleischmann, H. H., R. A. Young, and J. W. McGowan, Differential charge-transfer cross section for collisions of $\mathrm{H}^{+}$on $\mathrm{O}_{2}$, Phys. Rev., 153, 19-22, 1967.

Fleischmann, H. H., C. F. Barnett, and J. A. Ray, Small-angle scattering in stripping collisions of hydrogen atoms having energies of 1-10 keV in various gases, Phys. Rev. A, 10, 569583,1974

Galand, M., J. Lilensten, W. Kofman, and R. B. Sidje, Proton transport model in the ionosphere 1. Multistream approach of the transport equations, J. Geophys. Res., 102, 22261-22272, 1997.

Gao, R. S., L. K. Johnson, C. L. Hakes, K. A. Smith, and R. F. Stebbings, Collisions of kilo-electron-volt $\mathrm{H}^{+}$and $\mathrm{He}^{+}$with molecules at small angles: absolute differential cross sections for charge transfer, Phys. Rev. A, 41, 5929-5932, 1990.

Hedin, A. E., Extension of the MSIS thermosphere model into the middle and lower atmosphere, J. Geophys. Res., 96, 1159-1172, 1991.

Hultqvist, B., The hot ion component of the magnetospheric plasma and some relations to the electron component observations and physical implications, Space Sci. Rev., 23, 581-675, 1979.

Jasperse, J. R., Transport theoretic solutions for the beamspreading effect in the proton-hydrogen aurora, J. Geophys. Res. Lett., 24, 1415-1418, 1997.

Jasperse, J. R., and B. Basu, Transport theoretic solutions for auroral proton and $\mathrm{H}$ atom fluxes and related quantities, $J$. Geophys. Res., 87, 811-822, 1982.

Johnson, L. K., R. S. Gao, K. A. Smith, and R. F. Stebbings, Absolute differential cross sections for very small angle scattering of $\mathrm{keV} \mathrm{H}$ and $\mathrm{He}$ atoms by $\mathrm{H}_{2}$ and $\mathrm{N}_{2}$, Phys. Rev. A, 38, 2794-2797, 1988.

Kozelov, B. V., Influence of the dipolar magnetic field on transport of proton-H atom fluxes in the atmosphere, Ann. Geophysicae, 11, 697-704, 1993.

Kozelov, B. V., and V. E. Ivanov, Monte Carlo calculation of proton-hydrogen atom transport in $\mathrm{N}_{2}$, Planet. Space Sci., 40, 1503-1511, 1992.

Kozelov, B. V., and V. E. Ivanov, Effective energy loss per electronion pair in proton aurora, Ann. Geophysicae, 12, 1071-1075, 1994.

Lorentzen, D. A., F. Sigernes, and K. Henriksen, Numerical calculations and observations of the energy degradation of auroral protons and associated Balmer emission profiles in the upper atmosphere, Soc. Photo-Opt Instrum. Eng., 2050, 60-67, 1993.

McNeal, R. J., and J. H. Birely, Laboratory studies of collisions of energetic $\mathrm{H}^{+}$and hydrogen with atmospheric constituents, Rev. Geophys. Space Phys., 11, 633-693, 1973.
Miller, J. R., and G. G. Shepherd, Rocket measurements of $H_{\beta}$ production in a hydrogen aurora, J. Geophys. Res., 74, 49874997, 1969.

Newman, J. H., Y. S. Chen, K. A. Smith, and R. F. Stebbings, Differential cross sections for scattering of 0.5-, 1.5-, and 5.0keV hydrogen atoms by $\mathrm{He}, \mathrm{H}_{2}, \mathrm{~N}_{2}$ and $\mathrm{O}_{2}, J$. Geophys. Res., 91, 8947-8954, 1986.

Rees, M. H., On the interaction of auroral protons with the Earth's atmosphere, Planet. Space Sci., 30, 463-472, 1982.

Rees, M. H., Physics and chemistry of the upper atmosphere, Cambridge university press, Cambridge, 1989.

Sharber, J. R., The continuous (diffuse) aurora and auroral-E ionization, Physics of space plasmas SPI Conf. Proc. Reprint Ser., 4, 115-140, 1981.

Sharp, R. D., D. L. Carr, and R. G. Johnson, Satellite observations of the average properties of auroral particle precipitations: latitudinal variations, J. Geophys. Res., 74, 4618-4630, 1969.

Shen, D., Proton transport and auroral optical emissions, Ph. D. thesis, Geophys. Institute, Univ. Alaska, Fairbanks, Alaska, 1993.

Sigernes, F., Estimation of initial auroral proton energy fluxes from Doppler profiles, J. Atmos. Terr. Phys., 58, 1871-1883, 1996.

Sigernes, F., D. Lorentzen, C. S. Deehr, and K. Henriksen, Calculation of auroral Balmer volume emission height profiles in the upper atmosphere, J. Atm. Terr. Phys., 56, 503-508, 1994a.

Sigernes, F., G. Fasel, C. S. Deehr, R. W. Smith, D. A. Lorentzen, L. T. Wetjen, and K. Henriksen, Proton aurora on the dayside, Geomagn. Aeron., 34, 69-75, 1994b.

Sivjee, G. G., and B. Hultqvist, Particle and optical measurements in the magnetic noon sector of the auroral oval, Planet. Space Sci., 23, 1597-1601, 1975.

Söraas, F., H. R. Lindalen, K. Mâseide, A. Egeland, T. A. Sten, and D. S. Evans, Proton precipitation and the $H_{\beta}$ emission in a postbreakup auroral glow, J. Geophys. Res., 79, 1851-1859, 1974.

Söraas, F., K. Mâseide, P. Torheim, and K. Aarsnes, Dopplershifted auroral $H_{\beta}$ emission: a comparison between observations and calculations, Ann. Geophysicae, 12, 1052-1064, 1994.

Stamnes, K., Analytic approach to auroral electron transport and energy degradation, Planet. Space Sci., 28, 427-441, 1980.

Urban, A., Measurements of low-energy auroral ions, Planet. Space Sci., 29, 1353-1365, 1981.

Van Zyl, B., and H. Neumann, $H_{\alpha}$ and $H_{\beta}$ emission cross sections for low-energy $\mathrm{H}$ and $\mathrm{H}^{+}$collisions with $\mathrm{N}_{2}$ and $\mathrm{O}_{2}, J$. Geophys. Res., 85, 6006-6010, 1980.

Van Zyl, B., H. Neumann, T. Q. Le, and R. C. Amme, $\mathrm{H}+\mathrm{N}_{2}$ and $\mathrm{H}+\mathrm{O}_{2}$ collisions: experimental charge-production cross sections and differential scattering calculations, Phys. Rev. A, 18, 506-516, 1978.

Vegard, L., Emission spectra of night sky and aurora, Reports of the Gassiot Committee, Phys. Soc. Lon., 82, 1948.

Yousif, F. B., J. Gedded, and H. B. Gilbody, Balmer $\alpha$ emission in collisions of $\mathrm{H}, \mathrm{H}^{+}, \mathrm{H}_{2}^{+}$, with $\mathrm{N}_{2}, \mathrm{O}_{2}$ and $\mathrm{H}_{2} \mathrm{O}, \mathrm{J}$. Phys. B, 19, 217-231, 1986. 